\title{
Influence of Hydrodynamic Interactions on Colloidal Crystallization
}

\author{
Michio Tateno $\odot,{ }^{1}$ Taiki Yanagishima $\odot,{ }^{2}$ John Russo $\odot,{ }^{3}$ and Hajime Tanaka $\odot^{1, *}$ \\ ${ }^{1}$ Department of Fundamental Engineering, Institute of Industrial Science, University of Tokyo, \\ 4-6-1 Komaba, Meguro-ku, Tokyo 153-8505, Japan \\ ${ }^{2}$ Department of Chemistry, University of Oxford, Oxford OX1 3QZ, United Kingdom \\ ${ }^{3}$ Department of Physics, Sapienza University, P.le Aldo Moro 5, 00185 Rome, Italy
}

(Received 6 August 2019; published 20 December 2019)

\begin{abstract}
One of the biggest unresolved problems in crystallization phenomena is the significant discrepancy in the nucleation rate between experiments and simulations even for the simplest liquid, i.e., the hard-sphere system. A popular explanation for this discrepancy is the neglect of hydrodynamic interactions (HI) in simulation studies. By comparing simulations with and without HI, we show that the long-time diffusive dynamics of the colloids is slowed down more rapidly by hydrodynamic lubrication effects with increasing volume fraction. We find that the kinetics of both nucleation and growth are controlled by this long-time diffusion and that it is possible to account for most of the effects of $\mathrm{HI}$ by rescaling with this timescale. Therefore, we conclude that $\mathrm{HI}$ is not the primary cause of the accelerated nucleation rates observed in experiments.
\end{abstract}

DOI: 10.1103/PhysRevLett.123.258002

Introduction.-Understanding crystal nucleation is one of the most important issues in a wide range of materials, from metals, alloys, pharmaceuticals, to even food products. Phenomenological theories such as classical nucleation theory qualitatively capture key trends in crystallization, yet no common consensus has been reached regarding a general understanding of how crystallization occurs [1,2]. In an effort to enhance our understanding, more recent studies have focused on identifying microscopic pathways to crystal formation, where investigators followed transformations at the single-particle level [3-10]. Colloidal suspensions have aroused great interest in this context as an experimental system that exhibits analogous thermodynamic behavior to atomic systems and allows observation using optical microscopy with single-particle resolution [11]. In particular, hardsphere colloids have been studied intensively as a fundamental model system showing a liquid-crystal transition, providing a microscopic picture of crystal birth and growth. Yet Auer and Frenkel [12] found that there was an enormous mismatch in the crystal nucleation rate between light scattering experiments [13-15] and numerical predictions based on umbrella sampling simulations [12]. Revealing the physical origin behind this huge discrepancy remains a key challenge [10,16-19], and may be considered a central issue for the condensed matter field $[1,2]$.

The discrepancy in the nucleation rate between experiments and simulations implies the possibility that there is an unexpected nucleation pathway that leads to the efficient formation of a large nucleus. For example, unlike in classical nucleation theory, three-dimensional observation by confocal microscopy has shown that nuclei with a critical size have a nonspherical shape on average [20]. A simulation study [21] also claims that it is important to consider both nucleus morphology and the crystal polymorphs present in the nucleus. Two-step features of crystallization $[4,5,7,22,23]$ have been widely considered both in experiments [22] and simulations [4,5,7,23,24]. Recent experiments in charged colloidal systems [25,26] have also studied the effect of precursors in the nucleation process. Other effects arising from nonideality in experiments have been pointed out: e.g., polydispersity in the size of colloids [27], gravity (or, density mismatch between colloids and a solvent) [28,29]. For a review, see Ref. [2].

However, there is a potentially significant effect that most simulations neglect: the hydrodynamic degrees of freedom of the solvent. As far as we know, simulation studies that take the hydrodynamic interactions (HI) between colloids into account are limited to the following two cases. The first is the work on hard-sphere colloids by Radu and Schilling [30], where they examined the effect of HI using multiple particle collision dynamics. They found that the nucleation rate increases with increased solvent viscosity, concluding that HI may speed up nucleation and help resolve the discrepancy between experiments and simulations. The second work is a numerical study of crystal growth for colloids interacting via a Yukawa potential by Roehm et al. [31] using a fluctuating lattice Boltzmann method, where they reported that HI slows down crystallization. These works reach opposing conclusions; thus, further investigation of the effect of $\mathrm{HI}$ is required.

In this Letter, we examine the effect of $\mathrm{HI}$ on the crystallization kinetics of hard-sphere colloids using a fluid particle dynamics (FPD) method based on a direct computation of the Navier-Stokes equation [32]. By combining this method with fluctuating hydrodynamics, we may 
correctly introduce Brownian motion while satisfying statistical mechanical consistency both for the colloids and the solvent $[33,34]$. The validity of the FPD method for colloidal phase separation has been demonstrated previously by a direct adjustable-parameter-free comparison of simulation results with single-particle level 3D observation of a colloidal suspension using confocal microscopy [35].

System description.-To clarify whether HI may in fact be the origin of the huge mismatch in the crystal nucleation rate between experiments and molecular dynamics simulations, we undertake a comparative study of the crystallization process both in the presence and absence of HI by employing carefully matched FPD and Brownian dynamics (BD) simulations (overdamped Langevin equation without HI), respectively [see Supplemental Material (SM) [36] for detailed procedures of FPD and BD methods]. In this study, we approximate the hard-sphere potential by a Weeks-Chandler-Andersen potential:

$$
\begin{aligned}
U(r) & =4 \epsilon\left[\left(\frac{\sigma}{r}\right)^{12}-\left(\frac{\sigma}{r}\right)^{6}+\frac{1}{4}\right] \quad \text { for } \frac{r}{\sigma}<2^{1 / 6} \\
& =0 \text { otherwise, }
\end{aligned}
$$

where $\epsilon$ is the parameter determining the steepness of the repulsive potential. We set $\beta \epsilon=40$ following Refs. [4,18]. $\sigma$ corresponds to the diameter of colloids. We determine the effective hard-sphere diameter $\sigma_{\mathrm{HS}}$ by the following relation [44-46]:

$$
\sigma_{\mathrm{HS}}=\frac{2^{1 / 6}}{(1+1 / \sqrt{\beta \epsilon})^{1 / 6}} \sigma .
$$

With this diameter, we define the volume fraction $\phi=\pi \sigma_{\mathrm{HS}}^{3} N / 6 V$, where $N$ and $V$ are the number of particles and the volume of the simulation box, respectively.

Verification of simulation methods.-In FPD, solid colloidal particles are treated as undeformable liquid droplets with high viscosity. The interaction force between particles is introduced via a body force acting on the fluid elements. The first step in our investigation is to establish a connection between the volume fractions of the "smoothed" colloids defined in the FPD simulations, and the hard spheres defined in BD simulations. We look for thermodynamic consistency between the two types of simulations. We first run direct coexistence simulations of a crystal slab in coexistence with a fluid at several volume fractions within the coexistence region. Thermodynamically stable states in a hard-sphere system are a liquid for $\phi<\phi_{f} \sim 0.494$ and a face-centered cubic (fcc) crystal state for $\phi>\phi_{m} \sim 0.545$ with a coexistence region between them [47]. In the coexistence region, the volume fraction determines the relative amount of crystal and liquid according to the lever rule. We run simulations in the coexistence window until a stable fraction is obtained. Figure 1(a) shows the fraction of crystalline particles in
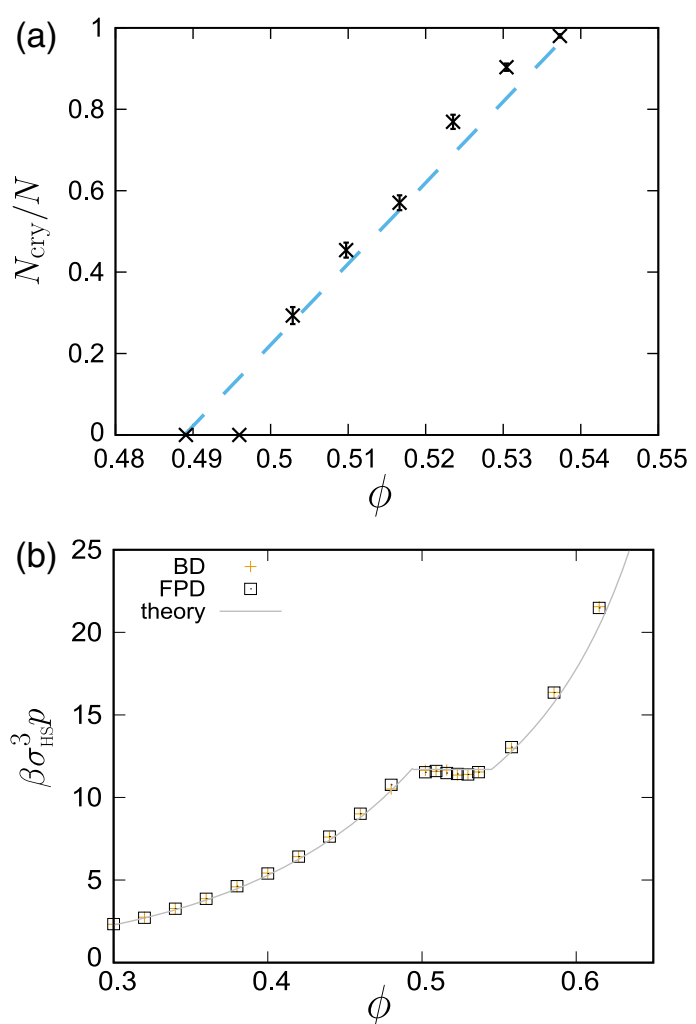

FIG. 1. Identification of liquid-crystal coexistence states. (a) Volume fraction $(\phi)$ dependence of the fraction of crystalline particles $N_{\text {cry }}$. The dashed line is what is expected from the lever rule, i.e., $\left(\phi-\phi_{f}\right) /\left(\phi_{m}-\phi_{f}\right)$. (b) Virial pressure as a function of volume fraction for BD and FPD. Separate theoretical predictions for the liquid and crystal branches and their coexistence are given as solid lines.

coexistence states as a function of the volume fraction $\phi$ (see Supplemental Material [36] for a detailed description of how the configurations were prepared). Our results fall on a straight line, as expected from the lever rule. The only exception is when the system is in close proximity to the melting and freezing points; this is simply because simulations cannot stabilize very thin layers of liquid or solid phases. Additionally, any displacement from the coexistence points (dashed line), is most likely due to the fact that there is some dependence of the fraction of crystalline particles on the order parameter thresholds used to distinguish crystal and liquid states (see Supplemental Material [36] for identification of crystals and their structures).

Having established a correspondence between the phase diagram of the FPD particles and those of hard spheres, we now focus on reproducing the thermodynamic properties of the stable phases, i.e., the liquid and the fcc crystal. To do this, we measure the osmotic pressure of equilibrium states using the virial pressure:

$$
p=\frac{N k_{B} T}{V}-\frac{1}{3 V} \sum_{i>j} r_{i j} \frac{\partial U\left(r_{i j}\right)}{\partial r_{i j}} .
$$


Figure 1(b) shows the volume fraction dependence of the virial pressure obtained by BD and FPD simulations. We see almost perfect agreement between the two simulations techniques, and with theoretical predictions using the Carnahan-Starling equation of state for the liquid phase, and the Speedy equation of state for the solid phase $[48,49]$. This strongly suggests the validity of investigating thermodynamic behavior using FPD.

Normalization of the kinetic factor controlling nucleation rate.-In dense suspensions, the motion of colloids significantly slows down due to the steric hindrance between colloids. However, the presence of the solvent suggests that the slowing down of colloid dynamics may also be brought about by the deformation of the solvent flow field between colloids. To examine the effects of $\mathrm{HI}$ on crystallization kinetics, we perform BD and FPD simulations and compare the resulting nucleation rates. To do so, it is crucial to consider the difference arising from the presence and absence of $\mathrm{HI}$ on particle transport. Here, it is worth mentioning that transport during crystal nucleation may generally be controlled by the long-time translational diffusion constant $D_{L}$ rather than the suspension viscosity $\eta$, or the structural relaxation time $\tau_{\alpha}$ [50]. This fact is important since the Stokes-Einstein relation is violated in a supercooled state: it is worth confirming this for colloidal systems.

In the inset of Fig. 2, we show the volume fraction dependence of the mean squared displacement (MSD), $\left\langle|\Delta \boldsymbol{R}|^{2}\right\rangle$, obtained by FPD simulations. We can see that the diffusive motion of colloids is suppressed as the volume

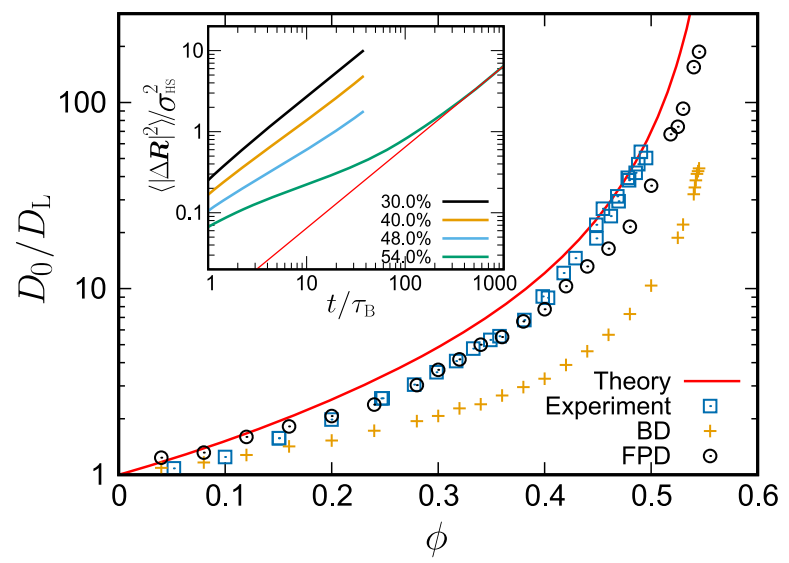

FIG. 2. Volume fraction dependence of long-time diffusion constant $D_{L}$ scaled by the diffusion constant in the dilute limit $D_{0}$. Crosses and circles represent results obtained using BD and FPD, respectively. The light blue squares are experimental data obtained by dynamic light scattering for hard-sphere like colloids with $5 \%$ polydispersity [51]. The red curve is a theoretical prediction that takes HI into account (see Ref. [52]). Inset: The mean square displacement for various volume fractions. Here, $\tau_{B}$ stands for the Brownian time of a free particle $\left(\tau_{B}=\sigma_{\mathrm{HS}}^{2} / 6 D_{0}\right)$. The red straight line represents the long-time asymptotic diffusion behavior. Here, we only show the results from FPD simulations. fraction increases. At $\phi=0.54$, we can see that the MSD shows a nonlinear dependence at early times and asymptotically approaches a linear trend. This behavior is a consequence of the caging effect, which is a common feature of supercooled liquids. We determined the long time diffusion coefficient $D_{L}$ by fitting the long time behavior of the MSD with $\left\langle|\Delta \boldsymbol{R}|^{2}\right\rangle=6 D_{L} t$. Figure 2 shows how the ratio between the single-particle diffusion coefficient $D_{0}$ and the long-time diffusion coefficient $D_{L}$ varies with volume fraction for both BD and FPD. We see that the long-term diffusion coefficient obtained with our FPD method (black circles) is consistent with results from both experiments (blue squares [51]) and theoretical calculations (red line [52]). For the BD method instead, we see a significant deviation from the other results, and that this difference becomes more significant at large $\phi$. For example, we see that $D_{L}$ in BD is approximately 4 times larger than that in FPD at $\phi=0.54$. This deviation originates from the presence of many-body HI.

Determination of nucleation rate via brute force simulations.-We will now consider the effect of $\mathrm{HI}$ on nucleation rates. We proceed to perform $\mathrm{BD}$ and FPD simulations for a range of volume fractions $0.54 \leq \phi \leq$ 0.545 . The system size is set to $L / \sigma_{\mathrm{HS}}=15.8$, with a particle number $N \sim 4000$ for the above volume fractions. According to a study using BD simulation [4], there are few finite-size effects on the nucleation rate with the above simulation size.

To determine the nucleation rate, we observe the time evolution of colloidal suspensions in a metastable liquid state at time $t=0$, and monitor the number of crystal nuclei $N_{c}(t)$. Here, we regard a crystal cluster as a crystal nucleus if the size of the cluster is above 100. For BD and FPD simulations, we performed 16 independent simulations for each of the volume fractions $\phi=0.540,0.541, \ldots, 0.545$ and $\phi=0.540,0.5425,0.545$, respectively. For BD, we confirmed crystallization in all runs. On the other hand, we were unable to continue FPD simulations until all the runs crystallized due to computational cost (the simulation time for each run being approximately $\sim 3000 \tau_{B}$, where $\tau_{B}=$ $\sigma_{\mathrm{HS}}^{2} / 6 D_{0}$ is the Brownian time of a free particle). 2, 9, and 14 runs actually crystallized for $\phi=0.540,0.5425$, and 0.545 , respectively. Thus, we take the following procedure [4] to estimate the nucleation rate: denoting the average of $N_{c}$ as $\left\langle N_{c}\right\rangle$ over all 16 trajectories, we determine the nucleation rate $I$ for each of BD and FPD simulations, by fitting the data with $\left\langle N_{c}\right\rangle / V=I\left(t-t_{0}\right)$, where $V$ is the volume and $t_{0}$ is a fitting parameter.

We scale the nucleation rates with the long-time diffusion coefficient we obtained independently and compare the results with the molecular dynamics result by Filion et al. [53]. Figure 3(a) shows that, despite the big difference in long-time diffusion scaling with $\phi$, all nucleation rates collapse onto a single curve. $\tau_{L}$ used in this figure is the Brownian time of a particle at $\phi$, defined as $\tau_{L}(\phi)=\sigma_{\mathrm{HS}}^{2} / 6 D_{L}(\phi)$. 

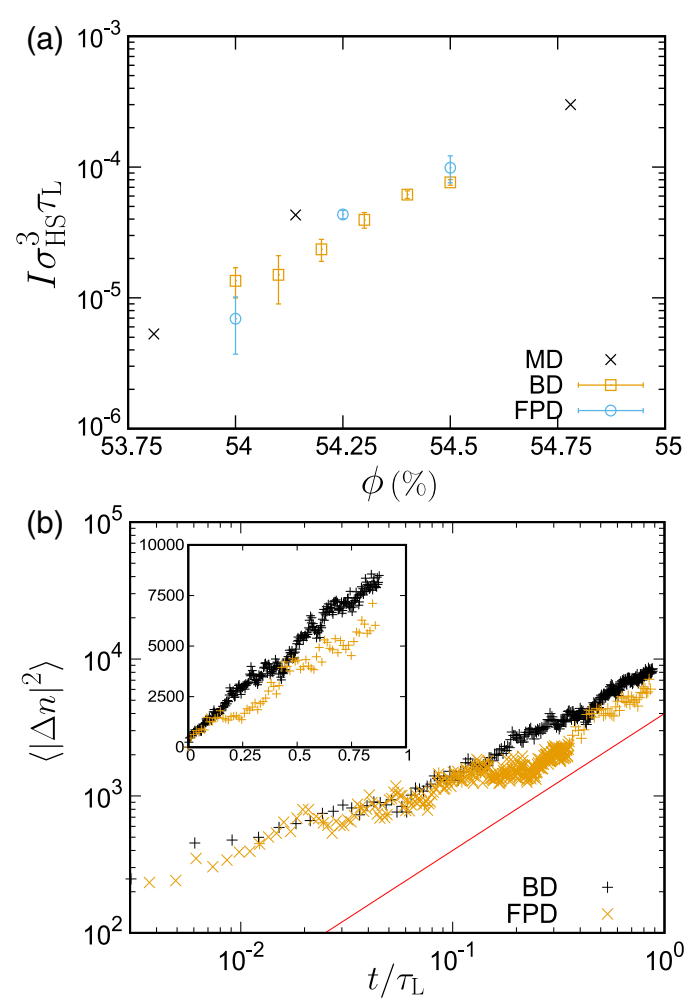

FIG. 3. (a) A comparison of crystal nucleation rates determined by BD and FPD methods. Rates found using molecular dynamics simulations (MD) by Filion et al. [53] are also shown. (b) Mean square displacement for nucleus size $\left\langle|\Delta n(t)|^{2}\right\rangle$ at the same volume fraction $\phi=0.5183$. The red line has a slope of 1 . The inset is the same data on a linear scale plot.

Time evolution of critical nucleus: estimation of kinetic factor.-Nucleation rates in hard spheres are known to change by many orders of magnitude within a small volume fraction window. Direct simulation results such as those reported in Fig. 3(a) can only access a small window around $\phi \sim 0.54$, where nucleation rates change by 2 orders of magnitude at most. The huge mismatch between simulation and experiments is instead more prominent at lower volume fractions $(\phi \sim 0.52$ or less).

To examine the behavior of nucleation rates at lower volume fractions, we employ the seeding technique [54], preparing a critical nucleus in the system and subsequently evolving it by BD and FPD simulations. By monitoring the growth or shrinkage of the nucleus, we can determine the kinetic factor that directly controls the nucleation rate. Following this strategy, we studied the time evolution of critical nuclei at $\phi=0.5183$, where the discrepancy between numerical and experimental nucleation rates is significantly larger (at least 8 orders of magnitude). To prepare a nucleus with a critical size, we first calculate the free energy barrier $\Delta F(n)$ ( $n$ being the size of the nucleus) using an umbrella sampling method (see Supplemental Material [36] for the details). Figure S2 shows the results when the critical size is set to $n_{c}=458$. We can indeed see that the maximum of $\Delta F$ is located near $n=458$. The height of the barrier, $\beta \Delta F\left(n_{c}\right)$, and the second derivative, $\beta \Delta F^{\prime \prime}\left(n_{c}\right)$, obtained by fitting are 70.6 and $-3.3 \times 10^{-4}$, respectively. We used ten independent initial configurations with a nucleus of $n=458$ and ran ten independent simulations of each (thus 100 runs in total). We then measured the number of runs that reached a crystalline basin in the energy landscape; the proportion that crystallized was 0.40 . The same procedure was repeated with a nucleus size of $n=465$; now, the ratio was 0.52 . Given its proximity to 0.50 , we consider $n=465$ to be the critical nucleus size and proceed to consider the time evolution of nuclei with 465 particles.

We performed BD and FPD simulations for 12 independently formed nuclei with 465 particles. Four independent runs were performed for each nucleus (48 runs in total), looking at the MSD in the nucleus size $\left\langle|\Delta n(t)|^{2}\right\rangle$ where $\Delta n(t)=n(t)-n(0)$. Figure 3(b) shows that $\left\langle|\Delta n(t)|^{2}\right\rangle$ increases linearly over time. Since $n(0) \sim n_{c}$, we can expect $\left\langle|\Delta n(t)|^{2}\right\rangle \sim 2 f_{+}\left(n_{c}\right) t$, where $f_{+}(n)$ is the transition rate for how quickly a nucleus of size $n$ gains a particle (see Supplemental Material [36] on the detail) $[12,55]$. Now, the key question here is what timescale these kinetic pathways should be scaled by. A natural candidate is the long-time diffusion time $\tau_{L}$. If we use this diffusion time $\tau_{L}$ and proceed to determine a scaled gradient, we find $f_{+}\left(n_{c}\right) \tau_{L} \sim 1.0 \times 10^{4}$ and $0.8 \times 10^{4}$ for $\mathrm{BD}$ and FPD, respectively. Noting that $\tau_{L}$ is the characteristic time of particle transport via translational diffusion, we see that rescaling the kinetic prefactor by $\tau_{L}$ effectively resolves the discrepancy in the nucleation rate between $\mathrm{BD}$ and FPD, as we also previously confirmed at a higher volume fraction $(\phi>0.54)$. This fact is a clear indication that HI, as implemented here, cannot account for the discrepancy between experiments and simulations.

Conclusion.-In conclusion, our work considers the effects of hydrodynamic interactions (HI) on the process of colloidal crystallization. We design a direct numerical simulation of the Navier-Stokes equations in which particles are embedded inside a viscous fluid as ultraviscous droplets that interact with a steep repulsive potential. We demonstrate that our methodology can reproduce the thermodynamic properties of the one-component hardsphere system with high accuracy. Nucleation and growth are both activated processes whose kinetic prefactor depends on the dynamics of the colloidal particles at the liquid-crystal interface. We consider nucleation at both high volume fraction $(\phi \sim 0.54)$, where it occurs spontaneously in our simulations, and at low volume fraction $(\phi \sim 0.52)$ with a seeding technique. Our results show that the dynamics of the colloidal particles changes more abruptly with HI interactions compared to the one component hard-sphere system usually adopted in simulations. Nevertheless, nucleation rates for all considered dynamics (FPD, BD, MD) collapse on the same curve when scaled by 
the long-time diffusion coefficient of the colloidal particles in the supercooled liquid. Long-time diffusion is thus the correct factor that controls the kinetic prefactor in nucleation; $\mathrm{HI}$ interactions can be accounted for with this single parameter. In other words, it is possible to account for most of the effects of HI by rescaling with the long-time diffusion time: this rules out $\mathrm{HI}$ from possible explanations for the huge discrepancy in the nucleation rate between experiments and simulations. The discrepancy still stands as one of the most interesting unresolved problems in soft condensed matter.

We thank Ryohei Shimizu for his contribution in the initial stage of this project. We are also grateful to Kyohei Takae and ISSP at the University of Tokyo for support in realizing a GPGPU implementation of the FPD method. The numerical simulations were performed on the SGI ICE $\mathrm{XA} / \mathrm{UV}$ hybrid system at the ISSP in the University of Tokyo. This study was partially supported by Grants-in-Aid for Specially Promoted Research (Grant No. JP25000002) and Scientific Research (A) (JP18H03675) from the Japan Society for the Promotion of Science (JSPS). T. Y. acknowledges a Grant-in-Aid for JSPS Research Fellows (16J06649). J. R. acknowledges support from the European Research Council Grant No. DLV-759187. M. T. acknowledges University of Tokyo Edge Capital and Roel Dullens for supporting his stay in the U.K.

*tanaka@iis.u-tokyo.ac.jp

[1] T. Palberg, J. Phys. Condens. Matter 26, 333101 (2014).

[2] J. Russo and H. Tanaka, J. Chem. Phys. 145, 211801 (2016).

[3] E. Zaccarelli, C. Valeriani, E. Sanz, W. C. K. Poon, M. E. Cates, and P. N. Pusey, Phys. Rev. Lett. 103, 135704 (2009).

[4] T. Kawasaki and H. Tanaka, Proc. Natl. Acad. Sci. U.S.A. 107, 14036 (2010).

[5] T. Schilling, H. J. Schöpe, M. Oettel, G. Opletal, and I. Snook, Phys. Rev. Lett. 105, 025701 (2010).

[6] E. Sanz, C. Valeriani, E. Zaccarelli, W. C. K. Poon, P. N. Pusey, and M.E. Cates, Phys. Rev. Lett. 106, 215701 (2011).

[7] J. Russo and H. Tanaka, Sci. Rep. 2, 505 (2012).

[8] E. Sanz, C. Valeriani, E. Zaccarelli, W. C. Poon, M. E. Cates, and P. N. Pusey, Proc. Natl. Acad. Sci. U.S.A. 111, 75 (2014).

[9] T. Yanagishima, J. Russo, and H. Tanaka, Nat. Commun. 8, 15954 (2017).

[10] N. Wood, J. Russo, F. Turci, and C. P. Royall, J. Chem. Phys. 149, 204506 (2018).

[11] B. Li, D. Zhou, and Y. Han, Nat. Rev. Mater. 1, 15011 (2016).

[12] S. Auer and D. Frenkel, Nature (London) 409, 1020 (2001).

[13] K. Schätzel and B. J. Ackerson, Phys. Rev. E 48, 3766 (1993).

[14] Y. He, B. J. Ackerson, W. van Megen, S. M. Underwood, and K. Schätzel, Phys. Rev. E 54, 5286 (1996).

[15] J. L. Harland and W. Van Megen, Phys. Rev. E 55, 3054 (1997).
[16] C. Sinn, A. Heymann, A. Stipp, and T. Palberg, in Trends in Colloid and Interface Science XV (Springer, New York, 2001), pp. 266-275.

[17] S. Iacopini, T. Palberg, and H. J. Schöpe, Phys. Rev. E 79, 010601(R) (2009).

[18] L. Filion, R. Ni, D. Frenkel, and M. Dijkstra, J. Chem. Phys. 134, 134901 (2011).

[19] M. Franke, A. Lederer, and H. J. Schöpe, Soft Matter 7, 11267 (2011).

[20] U. Gasser, E. R. Weeks, A. Schofield, P. Pusey, and D. Weitz, Science 292, 258 (2001).

[21] D. Moroni, P. R. ten Wolde, and P. G. Bolhuis, Phys. Rev. Lett. 94, 235703 (2005).

[22] H. J. Schöpe, G. Bryant, and W. van Megen, Phys. Rev. Lett. 96, 175701 (2006).

[23] T. Kawasaki and H. Tanaka, J. Phys. Condens. Matter 22, 232102 (2010).

[24] J. Russo and H. Tanaka, Soft Matter 8, 4206 (2012).

[25] P. Tan, N. Xu, and L. Xu, Nat. Phys. 10, 73 (2014).

[26] Y. Lu, X. Lu, Z. Qin, and J. Shen, Solid State Commun. 217, 13 (2015).

[27] S. Auer and D. Frenkel, Nature (London) 413, 711 (2001).

[28] J. Russo, A. C. Maggs, D. Bonn, and H. Tanaka, Soft Matter 9, 7369 (2013).

[29] S. Ketzetzi, J. Russo, and D. Bonn, J. Chem. Phys. 148, 064901 (2018).

[30] M. Radu and T. Schilling, Europhys. Lett. 105, 26001 (2014).

[31] D. Roehm, S. Kesselheim, and A. Arnold, Soft Matter 10, 5503 (2014).

[32] H. Tanaka and T. Araki, Phys. Rev. Lett. 85, 1338 (2000).

[33] A. Furukawa and H. Tanaka, Phys. Rev. Lett. 104, 245702 (2010).

[34] A. Furukawa, M. Tateno, and H. Tanaka, Soft Matter 14, 3738 (2018).

[35] M. Tateno and H. Tanaka, npj Comput. Mater. 5, 40 (2019).

[36] See Supplemental Material at http://link.aps.org/ supplemental/10.1103/PhysRevLett.123.258002 for identification of crystal structures, equilibrium states for measuring virial pressure, free energy barrier, and FPD method, which includes Refs. [37-43].

[37] P. J. Steinhardt, D. R. Nelson, and M. Ronchetti, Phys. Rev. B 28, 784 (1983).

[38] W. Lechner and C. Dellago, J. Chem. Phys. 129, 114707 (2008).

[39] W. Mickel, S. C. Kapfer, G. E. Schröder-Turk, and K. Mecke, J. Chem. Phys. 138, 044501 (2013).

[40] P. R. ten Wolde, M. J. Ruiz-Montero, and D. Frenkel, Phys. Rev. Lett. 75, 2714 (1995).

[41] P. R. ten Wolde, M. J. Ruiz-Montero, and D. Frenkel, J. Chem. Phys. 104, 9932 (1996).

[42] G. M. Torrie and J. P. Valleau, J. Comput. Phys. 23, 187 (1977).

[43] J. Russo, F. Romano, and H. Tanaka, Nat. Mater. 13, 733 (2014).

[44] D. Ben-Amotz and G. Stell, J. Phys. Chem. B 108, 6877 (2004).

[45] D. M. Heyes and H. Okumura, J. Chem. Phys. 124, 164507 (2006).

[46] A. Ahmed and R. J. Sadus, Phys. Rev. E 80, 061101 (2009). 
[47] W. G. Hoover and F. H. Ree, J. Chem. Phys. 49, 3609 (1968).

[48] R. J. Speedy, J. Phys. Condens. Matter 9, 8591 (1997).

[49] R. J. Speedy, J. Phys. Condens. Matter 10, 4387 (1998).

[50] H. Tanaka, Eur. Phys. J. E 35, 113 (2012).

[51] P. N. Segre, S. P. Meeker, P. N. Pusey, and W. C. K. Poon, Phys. Rev. Lett. 75, 958 (1995).
[52] M. Tokuyama and I. Oppenheim, Phys. Rev. E 50, R16 (1994).

[53] L. Filion, M. Hermes, R. Ni, and M. Dijkstra, J. Chem. Phys. 133, 244115 (2010).

[54] J. R. Espinosa, C. Vega, C. Valeriani, and E. Sanz, J. Chem. Phys. 144, 034501 (2016).

[55] S. Auer and D. Frenkel, J. Chem. Phys. 120, 3015 (2004). 